\title{
Diagnostic Bias in the COVID-19 Pandemic: A Series of Short Cases
}

\author{
Anete Zieda, Silvia Sbardella, Malika Patel, Richard W Smith \\ Milton Keynes University Hospital, Milton Keynes, UK
}

Received: 20/04/2021

Accepted: 25/04/2021

Published: $11 / 05 / 2021$

How to cite this article: Zieda A, Sbardella S, Patel M, Smith RW. Diagnostic bias in the COVID-19 pandemic: a series of short cases. EJCRIM 2021;8: doi:10.12890/2021_002575.

Conflicts of Interests: The authors declare there are no competing interests.

This article is licensed under a Commons Attribution Non-Commercial 4.0 License

\section{ABSTRACT}

During the COVID-19 pandemic, healthcare systems have faced unprecedented pressures. One challenge has been to promptly recognise non-COVID-19 conditions. Cognitive bias due to the availability heuristic may cause difficulties in reaching the correct diagnosis. Confirmation bias may also affect imaging interpretation. We report three cases with an alternative final diagnosis in whom COVID-19 was initially suspected: (a) Pneumocystis jirovecii pneumonia with unrecognised HIV infection; (b) pulmonary lymphangitis carcinomatosis; and (c) ST elevation myocardial infarction causing acute pulmonary oedema. To help mitigate bias, there is no substitute for thoughtful clinical assessment and critical appraisal when evaluating new information and formulating the differential diagnosis.

\section{LEARNING POINTS}

- The availability heuristic during the recent pandemic may lead to cognitive bias in favour of COVID-19 diagnosis and delayed recognition of other conditions, especially in patients presenting with similar non-specific features.

- Confirmation bias towards COVID-19 can also affect the interpretation of pulmonary imaging which is central to the investigation of cases with suspected pneumonitis.

- Diagnostic bias can be mitigated by recognition and allowing time for a thorough clinical history and methodical examination of the patients.

\section{KEYWORDS}

COVID-19, availability heuristic, cognitive bias, diagnostic bias

\section{CASE DESCRIPTIONS}

Case 1

A 46-year-old man presented with dyspnoea at rest, dry cough and malaise. He reported living with a family member with confirmed COVID-19. On examination, he was febrile with a $2 \mathrm{l} / \mathrm{min}$ oxygen requirement, and there were inspiratory crackles throughout both lungs. Chest x-ray (CXR) showed extensive bilateral air space shadowing (Fig. 1) and blood test results supported a provisional diagnosis of viral pneumonitis. Triaged to the COVID-19 admissions area, treatments were commenced accordingly. Pulmonary embolism was excluded on computed tomography pulmonary angiogram (CTPA) which showed widespread diffuse pulmonary changes, but notably also reported mediastinal lymphadenopathy and splenomegaly. Subsequently, the patient described a weight loss of $5 \mathrm{~kg}$ over 2 months. Examination revealed a slim build with a body mass index of 19 , oral leucoplakia and small raised violaceous cutaneous lesions over the trunk. Given these findings, HIV infection was strongly suspected. The patient was moved out of the open ward area into a side room. Despite the patient reporting no risk factors for retroviral infection, the result of HIV serology was positive. Intravenous co-trimoxazole was given for suspected Pneumocystis jirovecii pneumonia (PCP), later confirmed on sputum polymerase chain reaction (PCR) testing. Under specialist HIV services, with an initial low CD4 count of 70 cells $/ \mathrm{mm}^{3}$, the patient has responded well to anti-retroviral therapy. His COVID-19 swab tests remained 
negative despite exposure to other ward patients and the risks of hospital-acquired cross-infection. On review of the CXR, it is noteworthy that the changes were predominantly perihilar and in keeping with PCP, unlike the patchy distribution seen in COVID-19 pneumonitis where changes may be more peripheral.

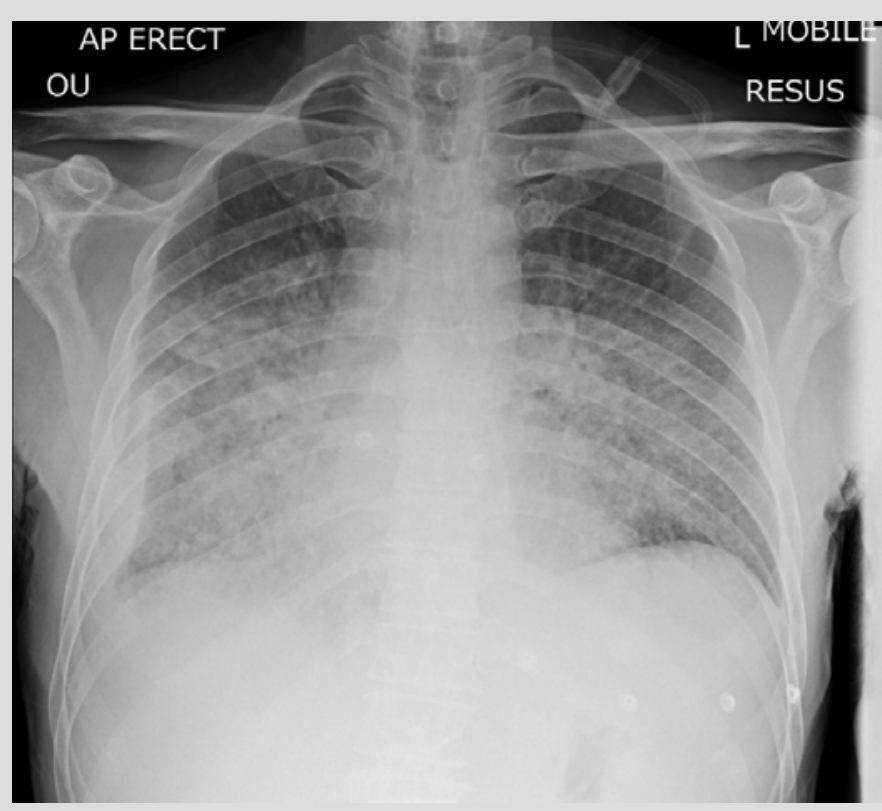

Figure 1. Chest $x$-ray in case 1 demonstrating extensive bilateral air space shadowing

Case 2

A 58-year-old woman presented with dyspnoea on minimal effort, tachypnoea and hypoxaemia at rest with saturations of $88 \%$ on room air. CXR demonstrated widespread reticular pattern abnormalities (Fig. 2). CTPA showed peripheral ground-glass changes suggestive of COVID-19 and widespread interlobular septal thickening (Fig. 3). A more detailed history from this patient revealed insidious and progressive dyspnoea over 4 weeks, reducing exercise tolerance, and weight loss. Repeat examination detected a $4 \mathrm{~cm}$ irregular and firm, non-mobile left supraclavicular mass, presumed to be a pathological lymph node. Urgent biopsy resulted in a diagnosis of metastatic adenocarcinoma of unknown primary. Following discussion at oncology multidisciplinary meetings, the imaging findings were ascribed to lymphangitis carcinomatosis.

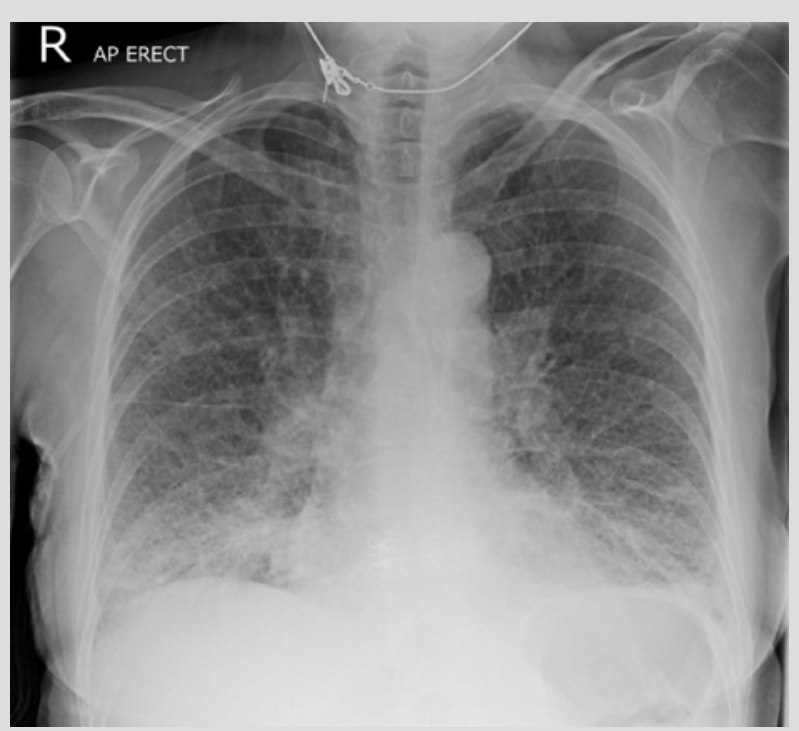

Figure 2. Chest $x$-ray in case 2 showing widespread reticular changes

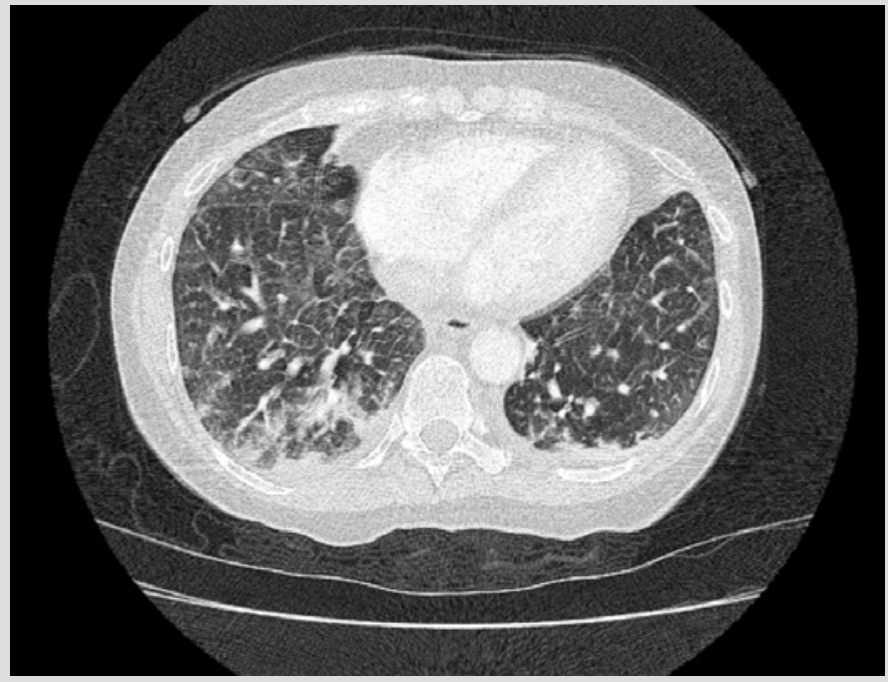

Figure 3. Computed tomography pulmonary angiogram in case 2 reported patchy groundglass opacification and interlobular septal thickening 
Case 3

A 62-year-old woman presented very unwell to the emergency department. She described acute severe retrosternal chest pain radiating through to her back. She was clammy and hypertensive, with oxygen saturations of $70 \%$ on breathing room air, and there were coarse bilateral inspiratory crackles on auscultation. She was transferred to the COVID-19 resuscitation bay. An urgent computed tomography aortogram (CT-aortogram) excluded suspected aortic dissection and pulmonary embolism. The scan was reported as probable COVID-19 pneumonitis (Fig. 4). An electrocardiogram (ECG) revealed marked inferior and anterior ST segment depression and T wave inversion. The initial troponin I was $40 \mathrm{ng} / \mathrm{ml}$. Remaining blood test results were surprisingly unremarkable. The working diagnosis remained COVID-19 pneumonitis, complicated by possible peri-myocarditis. The patient continued to report chest pain. Serial ECGs showed dynamic changes and the development of new significant ST segment elevation across the chest leads, suggesting an anterior ST elevation myocardial infarction. Urgent repeat troponin I was 2,211 ng/ml. Following emergency transfer for primary percutaneous coronary intervention, thrombotic occlusion of the proximal left anterior descending artery and significant stenosis of the mid-right coronary artery were stented successfully. The patient improved dramatically, and the lung changes were attributed to acute transient pulmonary oedema.

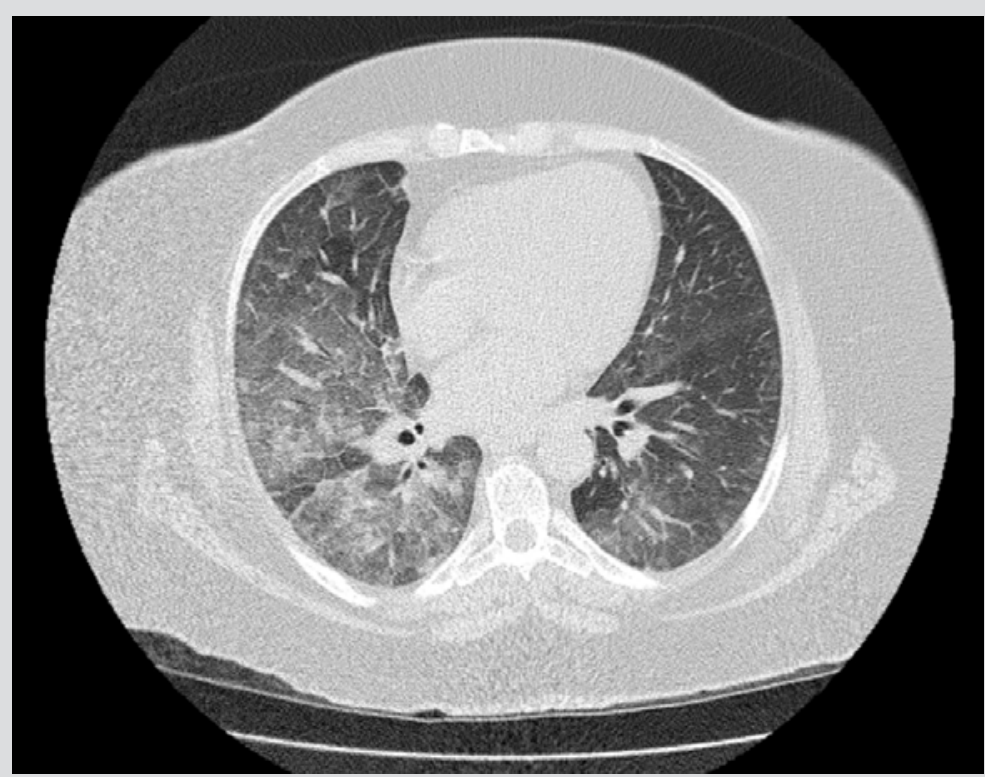

Figure 4. CT-aortogram in case 3 showing bilateral diffuse ground-glass opacification

\section{DISCUSSION}

During the COVID-19 pandemic, healthcare systems have struggled to deliver the standards of care expected normally ${ }^{[1]}$. Reduced rates of admission with acute cardiac and other conditions have been reported globally, attributed primarily to reluctance in seeking medical care associated with patients' fear of contracting COVID-19 at medical facilities and reduced accessibility to primary care and specialist services ${ }^{[1,2]}$. Having just emerged from the second wave of the pandemic, we are now seeing similar numbers of patients but a much larger variety of conditions being managed by our acute medical services in comparison to those during the most recent COVID-19 wave. In addition to previously reported factors contributing to decreased specialty admission rates during the pandemic, we suggest that the availability heuristic led to diagnostic bias towards COVID-19 and delays in the recognition of other acute presentations. Pulmonary imaging was central to the investigation in all reported cases, but here there was also evidence implicating a confirmation bias towards COVID-19 in interpretation and reporting. To help to mitigate the effects of bias, there is no substitute for a thoughtful clinical assessment and critical appraisal when evaluating new information.

\section{REFERENCES}

1. World Health Organization. Rapid assessment of service delivery for NCDs during the COVID-19 pandemic. 29 May 2020. https://www.who.int/who-documents-detail/ rapid-assessment-of-service-delivery-for-ncds-during-the-covid-19-pandemic (accessed 8 February 2021).

2. Aldujeli A, Hamadeh A, Briedis K, Tecson KM, Rutland J, Krivckas Z, et al. Delays in presentation in patients with acute myocardial infarction during the COVID-19 pandemic, Cardiol Res 2020;11(6):386-391. 